\section{Electroporation of proviral RCAS DNA alters gene expression in the embryonic chick hindbrain}

\author{
Petra M. Hermann and C. Cairine Logan \\ University of Calgary, Calgary, $A B$, Canada \\ BioTechniques 35:942-949 (November 2003)
}

\begin{abstract}
Gene transfer by means of electroporation is an effective method for delivering DNA into cells. Expression vectors encoding green fluorescent protein (GFP) are routinely used as a control for this technique and are also regularly used to indirectly or directly monitor the expression of introduced transgenes. However, recent studies suggest that GFP may have nonspecific and/or cytotoxic side effects. In this study, we investigated the effects of enhanced GFP (EGFP) expression delivered by means of electroporation of proviral RCASBP(B)-EGFP DNA on gene expression in the hindbrain of chick embryos. We examined, via whole-mount in situ hybridization, the expression of a number of transcription factors. We found that Tlx-1 was ectopically expressed following electroporation of proviral RCASBP(B)-EGFP DNA. In contrast, the number of cells expressing Tlx-3, Phox $2 \mathrm{a}$, and Phox $2 \mathrm{~b}$ were reduced. Intriguingly, these effects could be mimicked by electroporation of wild-type proviral RCASBP $(B) D N A$ (i.e., lacking the GFP insert). However, neither delivery of the EGFP transgene by means of viral infection nor electroporation alone yielded aberrant expression patterns. Together our data indicate that alterations of gene expression patterns are not directly due to the expression of EGFP but instead reflect a confounding effect of electroporating proviral DNA.
\end{abstract}

\section{INTRODUCTION}

Over the last decade, a variety of in vivo gene transfer techniques have been described and implemented. For instance, replication-competent retroviruses (e.g., RCAS vectors) (1) are now routinely used in the chick as an effective gene transfer system (2-4). Recently, electroporation, a relatively new gene transfer technique, has emerged as a leading technology for delivering DNA into both mitotic and postmitotic cells in vivo (5-10). Electroporation involves a transient permeabilization of the cellular plasma membrane by pulsed electric fields that allow exogenous polynucleotides to enter the cell. It has proven to be a very effective transfection technique, transferring multiple DNA copies into the cell. Nonviral expression vectors and, to a lesser extent, viral vectors (i.e., proviral DNA) have been used successfully to misexpress genes in a variety of organisms (10-12). Vectors encoding green fluorescent protein (GFP) or one of its mutant forms, such as enhanced GFP (EGFP), are routinely used as a control for this gene transfer technique. Interestingly, recent studies suggest that GFP and its analogs are not as biologically inert as previously thought (13-15). In this study, we examined the effects of in ovo electroporation of proviral RCASBP(B)-EGFP DNA on gene expression in the embryonic chick hindbrain.

\section{MATERIALS AND METHODS}

\section{Chick Embryos}

Fertilized Hubbard broiler hen eggs (i.e., not pathogen-free; IJtsma, Airdrie, AB, Canada) were incubated at $38^{\circ} \mathrm{C}$ for the times indicated. Embryos were staged according to the method of Hamburger and Hamilton (16).

\section{Retroviral Construction and Infection}

Chick embryo fibroblasts and concentrated viral stocks of the RCASBP(B) recombinant retroviral vector encoding EGFP [RCASBP(B)EGFP] were prepared and titered as described previously $(2,17)$. Concentrated viral supernatant (plus $80 \mu \mathrm{g} / \mathrm{mL}$ polybrene plus $0.01 \%$ Fast Green; Sigma, Oakville, ON, Canada) was injected using a picospritzer (Model PV820 Pneumatic Picopump; WPI, Sarasota, FL, USA) into the lumen of the neural tube of stage 7 to 8 embryos using glass micropipets (WPI) with an opening of 5-10 $\mu \mathrm{m}$. Howard's Ringer $(250 \mu \mathrm{L})$ containing penicillin/ streptomycin $(0.01 \%$; GIBCO/BRL, Burlington, ON, Canada) was carefully placed on top of the embryo immediately after each treatment. After injection, the eggs were sealed with Scotch tape and incubated for an additional $48 \mathrm{~h}$. The embryos were then fixed in $4 \%$ paraformaldehyde in phosphatebuffered saline (PFA/PBS), $\mathrm{pH} 7.4$, at $4^{\circ} \mathrm{C}$, examined under the fluorescent microscope for GFP, and subsequently processed via in situ hybridization.

\section{In Ovo Electroporation}

Proviral RCASBP(B)-EGFP DNA, a nonviral pCL-EGFP, or the wild-type proviral RCASBP(B) DNA (i.e., without the EGFP insert) were dissolved in Tris-EDTA (TE), pH 8.0, plus $0.01 \%$ Fast Green to approximately $1 \mu \mathrm{g} / \mu \mathrm{L}$ and injected in the lumen of the neural tube of stage 9 to 10 embryos. Subsequently, two L-shaped solid platinum electrodes $(0.2 \mathrm{~mm}$ diameter, $1 \mathrm{~mm}$ electrode length; WPI), positioned 4 $\mathrm{mm}$ apart, were placed on top of the vitelline membrane on either side of the hindbrain parallel to the neural tube. Care was taken to ensure that the electrodes did not touch the embryo. Ten 20 - to $25-\mathrm{V}$ square pulses $(50-65.5 \mathrm{~V} / \mathrm{cm})$ of $25 \mathrm{~ms}$ duration were generated at $1 \mathrm{~Hz}$ with an electroporator (Model ECM830 Electro Square Porator; BTX, San Diego, CA, USA). Because DNA is negatively charged, it migrates towards the anode during electroporation. Thus, due to the position of the electrodes, only one half of the hindbrain (the anode side) will be transfected. The contralateral half (the cathode side) acts as an internal control. Howard's Ringer $(250 \mu \mathrm{L})$ containing penicillin/streptomycin $(0.01 \%)$ was carefully placed on top of the embryo immediately after each operation. After electroporation, the eggs were sealed 
with Scotch tape and incubated for an additional $48 \mathrm{~h}$. The embryos were then fixed in $4 \%$ PFA/PBS at $4{ }^{\circ} \mathrm{C}$ and examined under the fluorescent microscope for green fluorescence signals.

\section{In Situ Hybridization}

Whole-mount RNA in situ hybridization was performed using nonradioactive digoxigenin (DIG)-labeled RNA probes as described previously (3), except that the embryos were hybridized at $74^{\circ} \mathrm{C}$. High-stringency posthybridization washes were also carried out at $74^{\circ} \mathrm{C}$, and the embryos were incubated at $4^{\circ} \mathrm{C}$ in pre-absorbed anti-DIG-alkaline phosphatase (AP) (Roche Diagnostics, Laval, Que, Canada) in TBST (137 mM NaCl, 2.7 mM KCL, 25 mM Tris-HCl, pH 7.5, 2 mM levamisol, and $1 \%$ Tween ${ }^{\circledR} 20$ ) for approximately 64 h. Specimens were refixed using $4 \%$ PFA/PBS prior to flat-mounting and/or storing. Antisense DIG-labeled RNA probes were synthesized according to the manufacturer's instructions (Roche Diagnostics) using the following DNA templates. For $T l x-1$ and $T l x-3,800-$ and 600-bp probes, entirely contained within their respective $3^{\prime}$-untranslated regions (UTRs), were made as previously described $(18,19)$. For Phox $2 a$ and $P$ hox $2 b$, gene-specific probes were made by linearizing pKSII Bluescript subclones containing partial Phox $2 a$ and Phox $2 b$ cDNA inserts of 389 and 374 bp, respectively, with EcoRI and transcribed using T7. For RCASBP(B), an approximately $1-\mathrm{kb} S a l \mathrm{I}$ to $\mathrm{ClaI}$ fragment from RCASBP(B) was subcloned into pSK Bluescript (Stratagene, La Jolla, CA, USA), with the resulting plasmid linearized using SalI and transcribed using T3.

\section{RESULTS}

To investigate whether the expression of EGFP transferred by means of electroporating proviral RCASBP(B) DNA affected gene expression patterns in the developing chick hindbrain, we focused our attention on the mRNA expression pattern of the divergent homeobox containing gene, Tlx-1 (also known as Hox11) $(18,20)$. Subsequently, to evaluate whether the effects described below were unique for $T l x-1$ or whether other genes were similarly affected, we also examined the expression of the closely related family member Tlx-3 (also known as Hox 1 1L2 and $R n x)(21,22)$ as well as two members of another family of transcription factors, Phox $2 a$ and Phox2b (23).

\section{Electroporation and Injection of Viral Expression Vectors}

$\begin{array}{clr}\text { Electroporation } & \text { of } & \text { proviral } \\ \text { RCASBP(B)-EGFP } & \text { DNA. } & \text { Injec- }\end{array}$ tion of the neural tube with proviral RCASBP(B)-EGFP DNA followed by electroporation resulted in distinct unilateral GFP expression in the hindbrain area in $88 \%$ of the preparations $(n=26)$. Since this procedure results in unilateral transfection of the hindbrain (i.e., only the "anode" side receives the transgene, indicated by red bar in Figure 1, A and $\mathrm{A}^{\prime}$ ), the other half of the hindbrain can be regarded as an internal control. Ectopic expression of $T l x-1$ was observed on the transfected side in $81 \%$ of the embryos expressing EGFP (Figure 1A'). In some embryos, ectopic expression of $T l x-1$ was restricted to a few rhombomeres, but in more than $60 \%$ of the embryos, ectopic Tlx -1 expression was observed in all rhombomeres (Figure 1A'). Interestingly, ectopic $T l x-1$ mRNA expression was restricted to the dorsal half of the neural tube (i.e., located dorsal to its normal domain of expression) (Figure 1 $\mathrm{A}^{\prime}$ ).

Infection with concentrated RCASBP(B)-EGFP viral supernatant. The above results show that the combination of proviral RCASBP(B)EGFP DNA and electroporation induced changes in the endogenous expression of $T l x-1$. To examine whether these effects were due to the method of delivery (i.e., electroporation) or due to the expression of the transgene itself, concentrated RCASBP(B)-EGFP supernatant (i.e., replication-competent retrovirus) was injected into the neural tube, and $T l x-1$ mRNA expression was examined. Infection with the replication-competent retrovirus resulted in widespread bilateral EGFP expression in the hindbrain in $80 \%$ of the embryos $(n=10)$ (Figure 1B). However, no ectopic expression of $T l x-1$ mRNA was observed in any of the retroviral infected embryos (Figure 1 $\mathrm{B}^{\prime}$ ). Thus, 
the ectopic $T l x-1$ expression observed following electroporation of the same vector is not simply due to expression of the transgene. The question remains however, is it is the electroporation technique itself or the combination of DNA transfer and electroporation that causes the aberrant $T l x-1$ expression.

Electroporation of the wild-type proviral RCASBP(B) DNA. To test the hypothesis that the electroporation procedure itself might be the cause of the aberrant $T l x-1$ mRNA expression following electroporation of proviral RCASBP(B) EGFP DNA, we injected the lumen of the neural tube with vehicle only (TE, $\mathrm{pH} 8.0$, plus $0.01 \%$ Fast Green) and subjected these preparations to electroporation protocols identical to those used above. Analysis of $T l x-1$ mRNA expression in these preparations revealed no ectopic $T l x-1$ mRNA in the hindbrain $(n=10$; data not shown).

To determine whether the electroporation procedure combined with the transfer of DNA induced the ectopic Tlx-1 mRNA expression, we injected and electroporated wild-type proviral $\operatorname{RCASBP}(\mathrm{B})$ DNA (i.e., lacking the inserted EGFP sequences) and subsequently analyzed $T l x-1$ mRNA expression. Electroporation with the wild-type proviral RCASBP(B) DNA resulted in unilateral expression of viral RNA in the hindbrain (Figure 1C). Ectopic Tlx-1 mRNA expression was detected in $88 \%$ of the embryos following electroporation of this vector $(n=$ 8; Figure $1 \mathrm{C}^{\prime}$ ). The expression pattern was remarkably similar to that observed following electroporation with the proviral RCASBP(B) DNA vector encoding EGFP (c.f., Figures 1, $\mathrm{A}^{\prime}$ and $\left.\mathrm{C}^{\prime}\right)$. Thus, we conclude that it is not the electroporation technique per se, but the combination of DNA transfer and electroporation that is responsible for the dramatic changes observed.

\section{Electroporation of a Nonviral Expression Vector}

To determine if the ectopic expression of $T l x-1$ was specific to the electroporation of proviral DNA expression vectors, we injected and electroporated a nonviral vector encoding EGFP (pCL-EGFP) into the hindbrain. GFP

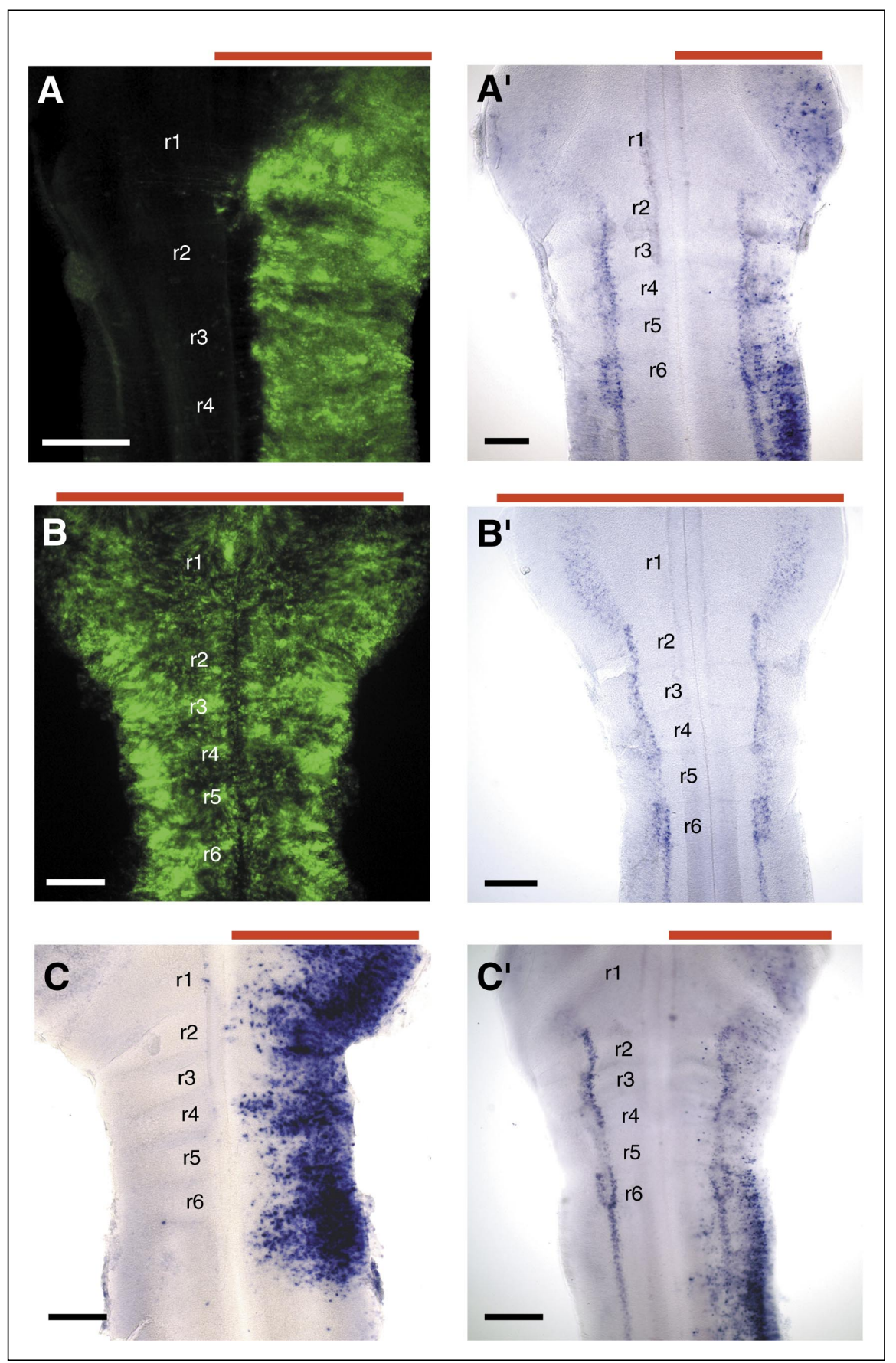

Figure 1. Gene expression determined by in situ hybridization after electroporation and injection of viral expression vectors. All photographs show dissected and flat-mounted hindbrains viewed from the ventricular surface. (A) Example of unilateral enhanced green fluorescent protein (EGFP) expression (green) in the hindbrain of a stage 23 embryo $48 \mathrm{~h}$ after neural tube injection and electroporation of RCASBP(B)-EGFP. (A') Ectopic Tlx-1 mRNA expression (blue) in a stage 21 embryo following neural tube electroporation of RCASBP(B)-EGFP. (B) Example of the widespread bilateral EGFP expression (green) in the hindbrain of a stage 21 embryo $48 \mathrm{~h}$ after infection with replication-competent retrovirus RCASBP(B)-EGFP. (B') Example of $T l x$-1 mRNA expression (blue) in a stage 21 embryo $48 \mathrm{~h}$ after infection with the retrovirus expressing EGFP. Note that the neural tube infection with replication-competent retrovirus RCASBP(B)-EGFP does not alter Tlx-1 mRNA expression in the hindbrain. (C) Example of unilateral RCASBP(B) expression (blue) in the hindbrain of a stage 22 embryo $48 \mathrm{~h}$ after neural tube injection and electroporation of wild-type proviral RCASBP(B) DNA. (C') Example of ectopic Tlx-1 mRNA expression (blue) in a stage 22 embryo following electroporation with the wild-type proviral RCASBP(B) vector. The site of DNA transfer (i.e., the anode site) in $\mathrm{A}, \mathrm{A}^{\prime}, \mathrm{C}$, and $\mathrm{C}^{\prime}$ and the infection areas in $\mathrm{B}$ and $\mathrm{B}^{\prime}$ are indicated by red bars. r1-r6 refers to rhombomere 1 to rhombomere 6 ; scale bars, $150 \mu \mathrm{m}$. 
fluorescence was readily detected unilaterally in the hindbrain following electroporation in $90 \%$ of the embryos $(n=10$; Figure 2A). However, no changes in Tlx- $1 \mathrm{mRNA}$ expression were observed (Figure 2A').

Together, our results indicate that the aberrant $T l x-1$ mRNA expression in the developing chick hindbrain is specific to electroporation of proviral DNA.

mRNA Expression of $T l x-3$, Phox 2a, and Phox $2 b$ Following Electroporation of Proviral DNA

To evaluate whether the effects described above are unique or whether electroporation of proviral RCASBP(B) DNA affects genes other than $T l x-1$, we examined the expression of the closely related family member Tlx-3 and two members of another family of transcription factors, Phox $2 a$ and Phox $2 b$. In contrast to $T l x-1$, the expression of $T l x-3$ was down-regulated in the hindbrain following injection and electroporation of either the proviral RCASBP(B)-EGFP DNA $(n=10)$ or the wild-type proviral RCASBP(B) DNA $(n=8)$. Figure $3 \mathrm{~A}$ shows the pattern of $T l x-3$ mRNA expression in the hindbrain following electroporation of proviral RCASBP(B) DNA. Although in some embryos a reduction in expression occurred in all rhombomeres, the effect was strongest in rhombomeres 1 and 2 (Figure 3A). Parenthetically, the apparent up-regulation of $T l x-3$ in rhombomere 4 was not observed in the other embryos. Overall, $28 \%$ of the embryos showed a distinct reduction in the expression of $T l x-3$ following electroporation with proviral RCASBP(B) DNA \pm EGFP. This effect was never observed following viral infection with the replication-competent retrovirus RCASBP(B)-EGFP (data not shown).

Interestingly, $71 \%-75 \%$ of the embryos injected and electroporated with the proviral RCASBP(B)-EGFP vector or the wild-type proviral RCASBP(B) DNA also showed a reduction in the number of cells expressing Phox $2 a$ or Phox $2 b$ ( $n=14$ and $n=8$, respectively). Figure 3B shows a clear reduction along the anterior-posterior axis in the number of cells expressing Phox $2 a$ in the transfected side. A similar effect was observed for Phox $2 b$ (data not shown).

\section{DISCUSSION}

In this study, we examined the effect of electroporation and retroviral-mediated gene transfer on the expression of endogenous genes in the hindbrain of embryonic chick. We showed that when a proviral, replication-competent DNA vector [RCASBP(B)-EGFP] was used in combination with electroporation to deliver a GFP transgene to the developing chick hindbrain, the expression of a number of transcription factors undergo marked changes. Electroporation of proviral RCASBP(B)-EGFP resulted in ectopic expression of $T l x-1$, while the number of cells expressing $T l x-3$, Phox $2 a$, and Phox $2 b$ were reduced. Significantly, similar changes in expression were seen when a wild-type proviral RCASBP(B) vector (i.e., lacking an insert) was used. However, neither delivery of the EGFP transgene by means of viral infection nor electroporation alone yielded aberrant expression patterns. This implies that the observed changes in gene

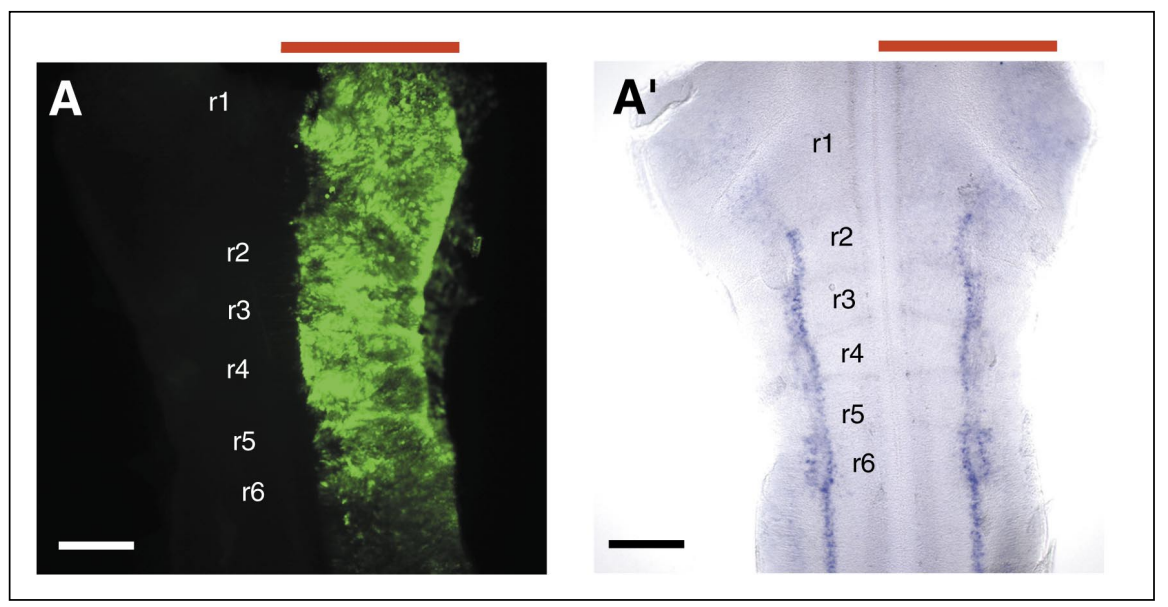

Figure 2. Gene expression determined by in situ hybridization after electroporation of a nonviral expression vector. All photographs show dissected and flat-mounted hindbrains viewed from the ventricular surface. (A) Example of unilateral enhanced green fluorescent protein (EGFP) expression (green) in the hindbrain of a stage 21 embryo $48 \mathrm{~h}$ after neural tube injection and electroporation of nonviral pCL-EGFP vector. (A') Example of $T l x-1$ mRNA expression (blue) in a stage 21 embryo $48 \mathrm{~h}$ after electroporation with the nonviral EGFP vector. The site of DNA transfer in A and $\mathrm{A}^{\prime}$ are indicated by the red bars. r1-r6 refers to rhombomere 1 to rhombomere 6; scale bars, $150 \mu \mathrm{m}$.

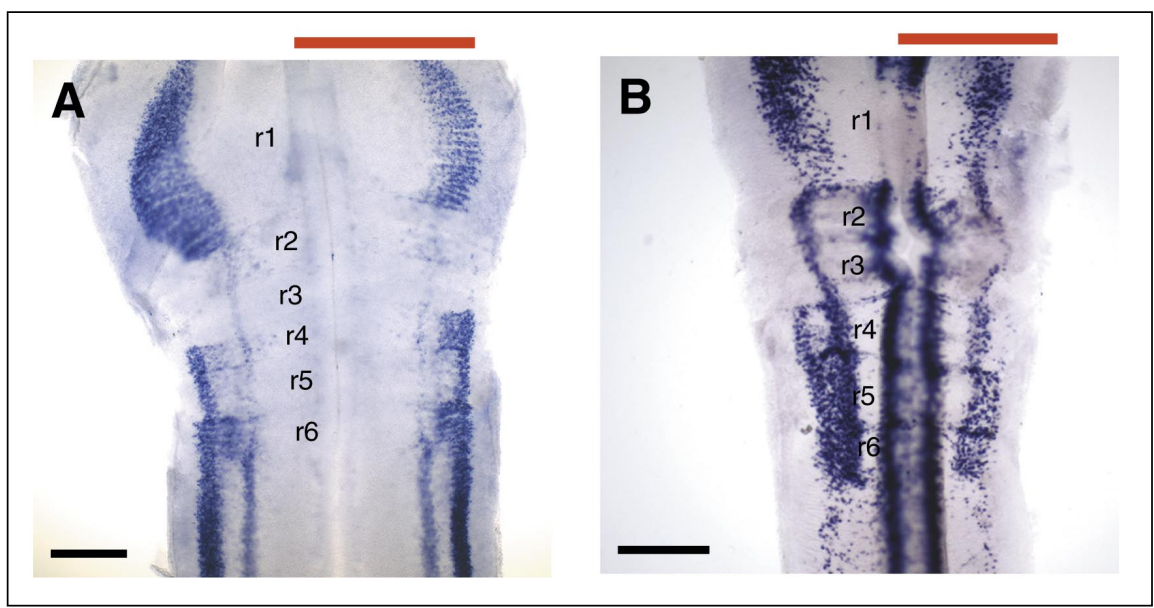

Figure 3. Gene expression determined by in situ hybridization after neural tube electroporation of RCASBP(B) DNA. The photographs show dissected and flat-mounted hindbrains viewed from the ventricular surface. (A) Example of the reduction in the number of $T l x-3$ expressing cells (blue) in rhombomere 1 of a stage 21 embryo following neural tube injection and electroporation of the proviral RCASBP(B) DNA. (B) Example of the reduction in the number of Phox2a expressing cells (blue) in the hindbrain of a stage 20 embryo following neural tube injection and electroporation of proviral RCASBP(B) DNA. The site of DNA transfer in A and B are indicated by the red bars. r1-r6 refers to rhombomere 1 to rhombomere 6 ; scale bars, $150 \mu \mathrm{m}$. 
expression were not simply due to the expression of EGFP itself, but rather reflect a confounding effect of electroporating a proviral RCASBP(B) DNA vector. At present, we can only speculate as to the explanation for this phenomenon. It is conceivable that the deleterious effects are associated with the replicative nature of the vector coupled with the high transfection efficiency of the electroporation method. Electroporating proviral DNA transfers multiple proviral copies into a cell. Theoretically, once inside the cell, the proviral DNA sequence is transcribed, and replication-competent retroviruses are produced. Proviral DNA sequences as well as replication-competent retroviruses have the potential to integrate into the genome (24-26). Thus, this method potentially results in the integration of multiple replication-competent viral copies in one cell, substantially increasing the risk of insertional mutagenesis. Interestingly, super infection of chick embryonic cells in culture has been shown to induce morphological and molecular abnormalities (27). In this respect, it is possible that different results may be obtained if a pathogen-free strain of embryos is used. In addition, the long terminal repeat (LTR) present in a variety of retroviruses (including Rous sarcoma) has been implicated in independently mediating trans activation of a variety of genes $(28,29)$. Thus, it is likely that the nature of the RCASBP(B) vector, combined with the high transfection efficiency of the electroporation method, may explain the aberrant gene expression.

\section{Specific Regions of Altered Gene Expression?}

We demonstrated that gene transfer by means of electroporation consistently resulted in unilateral expression of the transgene in the majority of the cells in the hindbrain. Intriguingly, we did not observe a 1:1 relationship between expression of the transgene and the ectopic expression of $T l x-1$. That is, while the transgene was uniformly expressed along the dorsal-ventral axis (i.e., from the most dorsal edge of the hindbrain to the ventral midline), ectopic $T l x-1$ expression was for the most part limited to dorsally located cells. Little or no ectopic expression was observed ventrally. Thus, only a subset of dorsally located cells express- ing the transgene ectopically expressed $T l x-1$. In this respect, it is interesting to note that the ectopic expression of $T l x-1$ tended to be located dorsal to its normal domain of expression. On the other hand, although unlikely, we cannot exclude that this distribution results from local variations in electrical field strength and/or vector distribution. Although cell death might explain the observed down-regulation in expression of $T l x-3$, Phox $2 a$, and $P h o x 2 b$, it is unlikely to explain the observed ectopic expression of $T l x-1$. Furthermore, if the electroporated cells were being selectively killed, transgene expression should also be lost. Clearly, the majority of the cells in the hindbrain still express the transgene $48 \mathrm{~h}$ after electroporation.

\section{Direct or Indirect Effects?}

One of the questions left to answer is how RCASBP(B) triggers the changes in expression of the four transcription factors reported here (i.e., are the effects due to direct or indirect effects on transcriptional regulation of each of the genes). In this context, it is interesting that the same treatment, which causes an up-regulation of $T l x-1$ expression, causes the expression of a second family member, $T l x-3$, to be down-regulated. Although it is possible that $T l x-1$ and Tlx-3 mutually repress each others' expression, as has been shown for class I and class II proteins $(11,30,31)$, this seems unlikely because we observed no overlap in the areas showing aberrant expression of $T l x-1$ and the areas in which $T l x-3$ expression was downregulated. Also, ectopic expression of Tlx -1 was observed in $80 \%-88 \%$ of the embryos, whereas $T l x-3$ was affected in only $28 \%$ of the embryos, suggesting that $\mathrm{RCASBP}(\mathrm{B})$ regulates expression of these two family members independently. In addition, although there seems to be overlap in expression patterns between the Tlx and the Phox family members $(18,23,32)$, a mouse $T l x-3$ knockout study indicated that the expression of Tlx-3 and the Phox family is independently regulated, and it seems unlikely that $T l x-3$ is a direct downstream target of Phox $2 a$ or Phox $2 b$ (32).

Overall, our data strongly suggests that regulatory mechanisms intercede in the effects of the provirus rather than some nonspecific mechanism enhancing gene transcription in a global fashion. Moreover, our study indicates that transferring proviral RCASBP(B) DNA by means of electroporation has widespread consequences on the expression of a multitude of genes during early embryogenesis.

\section{ACKNOWLEDGMENTS}

The authors wish to thank Dr. H. Rohrer (Max-Planck Institut fur Hirnforschung, Frankfurt, Germany) for kindly providing the chick Phox $2 \mathrm{a}$ and Phox2b mRNA riboprobes, Drs. H. Kurz (University of Freiburg, Germany) and J. Gilthorpe (King's College London, UK) for kindly providing the $p C L-E G F P$ and the RCASBP(B)-EGFP vectors, respectively, Kathy Rouleau for excellent technical assistance, and Drs. W.C. Wildering, G. van Marle, and C. Power for critically reading the manuscript. This research was supported by grants from the Canadian Institutes of Health Research (CIHR) and the Alberta Heritage Foundation for Medical Research Canada (AHFMR). C.C.L. is an AHFMR and CIHR scholar.

\section{REFERENCES}

1. Hughes, S.H., J.J. Greenhouse, C.J. Petropoulos, and P. Sutrave. 1987. Adaptor plasmids simplify the insertion of foreign DNA into helper-independent retroviral vectors. J. Virol. 61:3004-3012.

2. Fekete, D. and C.L. Cepko. 1993. Replication-competent retroviral vectors encoding alkaline phosphatase reveal spatial restriction of viral gene expression/transduction in the chick embryo. Mol. Cell. Biol. 13:2604-2613.

3. Logan, C., A. Wizenmann, E. Drescher, B. Monschau, F. Bonhoeffer, and A. Lumsden. 1996. Rostral optic tectum acquires caudal characteristics following ectopic engrailed expression. Curr. Biol. 6:1006-1014.

4. Edom-Vovard, F., M.A. Bonni, and D. Duprez. 2001. Misexpression of Fgf-4 in the chick limb inhibits myogenesis by down-regulating Frek expression. Dev. Biol. 233:56-71.

5. Muramatsu, T., Y. Mizutani, Y. Ohmori, and J. Okumura. 1997. Comparison of three nonviral transfection methods for foreign gene expression in early chicken embryos in ovo. Biochem. Biophys. Res. Comm. 230: 376-380.

6. Funahashi, J., T. Okafuji, H. Ohuchi, S. Noji, H. Tanaka, and H. Nakamura. 1999. Role of Pax-5 in the regulation of a mid-hindbrain organizer's activity. Dev. Growth Differ. 41:59-72. 
7. Nakamura, H., Y. Watanabe, and J. Funahashi. 2000. Misexpression of genes in brain vesicles by in ovo electroporation. Dev. Growth Differ. 42:199-201.

8. Atkins, R.L., D. Wang, and R.D. Burke. 2000. Localized electroporation: a method for targeting expression of genes in avian embryos. BioTechniques 28:94-96.

9. Somiari, S., J. Glasspool-Malone, J.J. Drabick, R.A. Gilbert, R. Heller, M.J. Jaroszeski, and R.W. Malone. 2000. Theory and in vivo application of electroporative gene delivery. Mol. Therapy 2:178-187.

10. Haas, K., W.-C. Sin, A. Javaherian, Z. Li, and H.T. Cline. 2001. Single-cell electroporation for gene transfer in vivo. Neuron 29: 583-591.

11. Vallstedt, A., J. Muhr, A. Pattyn, A. Pierani, M. Mendelsohn, M. Sander, T.M. Jessell, and J. Ericson. 2001. Different levels of repressor activity assign redundant and specific roles to Nkx6 genes in motor neuron and interneuron specification. Neuron 31: 743-755.

12. Yaneza, M., J.D. Gilthorpe, A. Lumsden, and A.S. Tucker. 2002. No evidence for ventrally migrating neural tube cells from the mid- and hindbrain. Dev. Dynam. 223: 163-167.

13. Liu, H.-S., M.-S. Jan, C.-K.Chou, P.-H. Chen, and N.-J. Ke. 1999. Is green fluorescent protein toxic to the living cells? Biochem. Biophys. Res. Comm. 260:712-717.

14. Felts, K., B. Rogers, K. Chen, H. Ji, J. Sorge, and P. Vaillancourt. 2001. Recombinant Renilla reniformis GFP displays low toxicity. Stratagene Newsletter 13:85-87.

15. Garner, S., T. Macatee, P. Vaillancourt, B. Rogers, and Y. Wang. 2001. Don't let a toxic GFP skew your results! Stratagene Newsletter 14:86-87.

16. Hamburger, V. and H.L. Hamilton. 1951. A series of normal stages in the development of the chick embryo. J. Morphol. 88:49-92.

17. Logan, C. and P. Francis-West. 1999. Gene transfer in avian embryos using replication competent retroviruses, p. 539-551. In P.T Sharpe and I. Mason (Eds.), Methods in Molecular Biology: Molecular Embryology: Methods and Protocols. Humana Press, Totowa.

18. Logan, C., R.J.T. Wingate, I.J. McKay, and A. Lumsden. 1998. Tlx-1 and Tlx-3 homeobox gene expression in cranial sensory ganglia and hindbrain of the chick embryo: markers of patterned connectivity. J. Neurosci. 18:5389-5402.

19. Logan, C., C. Millar, V. Bharadia, and K. Rouleau. 2002. Onset of Tlx-3 expression in the chick cerebellar cortex correlates with the morphological development of fissures and delineates a posterior transverse boundary. J. Comp. Neurol. 448:138-149.

20. Kennedy, M.A., R. Gonzalez-Sarmiento, U.R. Kees, F. Lampert, N. Dear, T. Boehm, and T.H. Rabbitts. 1991. HOX11, a homeobox-containing T-cell oncogene on human chromosome 10q24. Proc. Natl. Acad. Sci. USA 88:8900-8904.

21. Cheng, S.H. and T.W. Mak.1993. Molecular characterization of three murine HOX11-related homeobox genes, Tlx-1, -2 , and -3 , and restricted expression of Tlx-1 during embryogenesis. Dev. Growth Differ. 35:655-663.

22. Dear, T.N., I. Sanchez-Garcia, and T.H. Rabbitts. 1993. The HOX11 gene encodes a DNA-binding nuclear transcription factor belonging to a distinct family of homeobox genes. Proc. Natl. Acad. Sci. USA 90:44314435.

23. Pattyn, A., X. Morin, H. Cremer, C. Goridis, and J.F. Brunet. 1997. Expression and interactions of the two closely related homeobox genes Phox2a and Phox2b during neurogenesis. Development 124:4065-4075.

24. Whitcomb, J.M. and S.H. Hughes. 1992. Retroviral reverse transcription and integration: progress and problems. Annu. Rev. Cell Biol. 8:275-306

25. Kulkosky, J. and A.M. Skalka. 1994. Molecular mechanism of retroviral DNA integration. Pharmacol. Ther. 61:185-203.

26. Goff, S.P. 2001. Intracellular trafficking of retroviral genomes during the early phase of infection: viral exploitation of cellular pathways. J. Gene Med. 3:517-528

27. Colby, C. and H. Rubin. 1969. Growth and nucleic acid synthesis in normal cells and cells infected with Rous sarcoma virus. J. Natl. Cancer Inst. 43:437-444.

28. Faller, D.V., H. Weng, D.T. Graves, and S.Y. Choi. 1997. Moloney murine leukemia long terminal repeat activates monocyte chemotactic protein-protein expression and chemotactic activity. J. Cell. Physiol. 172: 240-252.

29. Ghosh, S.K. and D.V. Faller.1999. Feline leukemia virus long terminal repeat activates collegenase IV gene expression through AP-1. J. Virol. 73:4931-4940.

30. Briscoe, J., A. Pierani, T.M. Jessell, and J. Ericson. 2000. A homeodomain protein code specifies progenitor cell identity and neuronal fate in the ventral neural tube. Cell 101:435-445.

31. Sander, M., S. Paydar, J. Ericson, J. Briscoe, E. Berber, M. Germann, T.M. Jessell, and J.L. Rubenstein. 2000. Ventral neural patterning by Nkx homeobox genes: Nkx6.1 controls somatic motor neuron and ventral interneuron fates. Genes Dev. 17:2134-2139.

32. Qian, Y., B. Fritzsch, S. Shirasawa, C.L. Chen, Y. Choi, and Q. Ma. 2001. Formation of brainstem (nor)adrenergic centers and firstorder relay visceral sensory neurons is dependent on homeodomain protein Rnx/Tlx3. Genes Dev. 15:2533-2545.

Received 28 May 2003; accepted 23 July 2003.

Address correspondence to:

C. Cairine Logan

Genes and Development Research Group

Department of Cell Biology and Anatomy

Faculty of Medicine

University of Calgary

HSC, 3330 Hospital Drive, N.W.

Calgary, AB, T2N 4N1 Canada

e-mail:clogan@ucalgary.ca 